\title{
Antimony mobility and influence on iron(II)-catalyzed ferrihydrite transformation pathways
}

\author{
K. HOCKMANN ${ }^{1, *}$, N. KARIMIAN ${ }^{2}$, S. SCHLAGENHAUFF ${ }^{1}$,
} B. PlANER-FrIEDRICH ${ }^{1}$, E.D. BURTON ${ }^{2}$

${ }^{1}$ University of Bayreuth, Bayreuth Center for Ecology and Environmental Research, 95440 Bayreuth, Germany

(*correspondence: kerstin.hockmann@uni-bayreuth.de)

${ }^{2}$ Southern Cross University, Southern Cross GeoScience, Lismore NSW 2480, Australia

The mobility of antimony ( $\mathrm{Sb}$ ), a toxic metalloid of increasing environmental concern, is largely controlled by sorption to iron $(\mathrm{Fe})$ oxides. In wetland soils and sediments, $\mathrm{Fe}(\mathrm{II})$ may accelerate the transformation and crystallization of poorly ordered $\mathrm{Fe}$ oxides such as ferrihydrite to more stable phases. These changes in $\mathrm{Fe}$ oxide mineralogy can cause major shifts in the number of sites available for surface complexation and may thus affect the partitioning and bioavailability of associated $\mathrm{Sb}$.

Here, we investigated $\mathrm{Sb}$ partitioning during the $\mathrm{Fe}(\mathrm{II})$ induced transformation of Sb-bearing ferrihydrite across a range of $\mathrm{Sb}(\mathrm{V})$ loadings (molar ratios of $\mathrm{Sb}: \mathrm{Fe}=0.003,0.016$ and 0.08 ) at $\mathrm{pH}$ 7. Changes in Fe mineral assemblages and $\mathrm{Sb}$ sorption mechanisms were evaluated using extended X-ray absorption fine structure (EXAFS) spectroscopy, powder Xray diffraction (XRD) and wet chemical extraction techniques.

Addition of $\mathrm{Fe}(\mathrm{II})$ induced the rapid conversion of ferrihydrite via metastable lepidocrocite to goethite at low and medium $\mathrm{Sb}$ loadings. The highest $\mathrm{Sb}: \mathrm{Fe}$ ratio favored the formation of feroxyhyte from precursor ferrihydrite. The transformation of ferrihydrite was faster in the presence of $\mathrm{Sb}$ compared to no-Sb control treatments and was paralleled by a decrease in aqueous and phosphate-extractable $\mathrm{Sb}$ concentrations. EXAFS shell-fit analyses revealed that this $\mathrm{Sb}$ immobilization was attributable to the incorporation of $\mathrm{Sb}$ into the structure of the neo-formed Fe oxides.

Our results are important for a robust understanding of $\mathrm{Sb}$ geochemistry in redox-dynamic environments as they demonstrate that $\mathrm{Sb}$ itself influences the pathways and kinetics of $\mathrm{Fe}$ oxide transformations. This study also suggests that $\mathrm{Fe}$ oxide transformation pathways in Sb-contaminated systems may differ strongly from the well-known pathways that occur under Sb-free conditions. 